\title{
Circuit
}

Musiques contemporaines

\section{Réseaux des arts médiatiques : à l'écoute...}

\section{Barah Héon-Morissette}

Volume 22, numéro 3, 2012

URI : https://id.erudit.org/iderudit/1014231ar

DOI : https://doi.org/10.7202/1014231ar

Aller au sommaire du numéro

Éditeur(s)

Les Presses de l’Université de Montréal

ISSN

1183-1693 (imprimé)

1488-9692 (numérique)

Découvrir la revue

Citer cet article

Héon-Morissette, B. (2012). Réseaux des arts médiatiques : à l'écoute.... Circuit, 22(3), 73-76. https://doi.org/10.7202/1014231ar

Ce document est protégé par la loi sur le droit d'auteur. L'utilisation des services d’Érudit (y compris la reproduction) est assujettie à sa politique d'utilisation que vous pouvez consulter en ligne.

https://apropos.erudit.org/fr/usagers/politique-dutilisation/
Cet article est diffusé et préservé par Érudit.

Érudit est un consortium interuniversitaire sans but lucratif composé de l’Université de Montréal, l'Université Laval et l'Université du Québec à Montréal. Il a pour mission la promotion et la valorisation de la recherche. https://www.erudit.org/fr/ 


\section{Réseaux des arts médiatiques:}

\section{à l'écoute...}

Barah Héon-Morissette

\section{... panoramique}

Vu de loin, le panorama semble statique, figé dans une carte postale. Immuable, il ne reflète pas la marque du temps. Même le vent le plus furieux ne fait pas bouger le roc. L'image reste silencieuse. Vu de près, au centre du paysage, l'homme perçoit la moindre feuille frémir sous la brise. Chaque instant marque son passage, laisse une trace. Voilà deux manières de percevoir le temps qui passe. Déconcerté par la relation au temps? «zo ans? Qu'est-ce que 20 ans? »

Assurément, vingt ans pour un organisme culturel, c'est très respectable. D'autant plus lorsque nous parlons de création! Le journaliste Réjean Beaucage faisait la même remarque pour les quinze ans de cette institution montréalaise dans son «Portrait de Réseaux des arts médiatiques ». Cinq ans plus tard, faut-il encore s'arrêter au passage du temps? Réseaux est le miroir de l'environnement sonore actuel. Il s'adapte aux conditions, il grandit avec les tendances. Il vit!

Le paysage sonore montréalais a pris une autre couleur depuis la naissance de Réseaux des arts médiatiques, en 1991. Essentiel dans le tableau culturel, il est encore unique en son genre au Canada par le mandat qu'il s'est donné et respecte toujours: présenter les musiques électroacoustiques de concert. Les trois fondateurs Robert Normandeau, Jean-François Denis et Gilles Gobeil ont senti le besoin de faire connaître cette musique et surtout de donner les moyens aux artistes de diffuser leurs œuvres dans de meilleures conditions. À cet effet, à la veille de fonder Réseaux, Normandeau fait état de ses réflexions dans l'Espace du son II : «Il apparaît de plus en plus clairement aujourd'hui que pour rendre justice à cette notion d'espace, un certain nombre de conditions doivent être réunies notamment dans une situation de concert².» C'était dans l'air du temps!
1. Réjean Beaucage (2006), "Portrait de Réseaux des arts médiatiques", Circuit: musiques contemporaines, vol. 16, $\mathrm{n}^{\circ} 3$, p. 107.

2. Robert Normandeau (1991), "L'Espace en soi", in Francis Dhomont (dir.), Espace du son II, Ohain, Musiques \& Recherches, p. 115. 
3. Maxime McKinley (2006), "Ultime édition de Rien à voir: entretien avec Francis Dhomont sur l'avenir acousmatique", Circuit: musiques contemporaines, vol. 16, $n^{0} 3$, p. 120.

4. Francis Dhomont (2012), "Autour de l' "École de Montréal" ", communication présentée en collaboration avec Réseaux, Akousma@cıRmmt, Centre interdisciplinaire de recherche en musique, médias et technologie, Montréal (5 avril).

5. Aкousma à Rimouski, 26 mai 2010, et Akousma à Gatineau, 12 mai 2010.

\section{... renouvelée}

Pendant près de dix-sept années, la direction artistique est assurée par le trio des fondateurs. Initialement préoccupés par le développement de la culture acousmatique au Québec, ils présentent des compositeurs étrangers afin d'offrir au public québécois des œuvres d'envergure qui sont maintenant dans le catalogue du répertoire acousmatique. Après quinze éditions de la série de concerts Rien à voir et quelques incursions dans le chemin de la musique mixte, Réseaux des arts médiatiques sent le vent tourner et commence à emprunter une nouvelle voie. Alors que naît le Festival Akousma (janvier 2005) et que Rien à voir tire sa révérence (février 2004), Francis Dhomont, professeur de composition électroacoustique à l'Université de Montréal de 1980 à 1996, confie son inquiétude à Maxime McKinley dans une entrevue par correspondance: «[...] il me parait inopportun de quitter la route aujourd'hui, à un moment où - dans le contexte montréalais - elle semble provisoirement plus étroite, pour s'engager dans une voie plus large mais déjà très fréquentée3.» Avec le recul que procurent les années, il semble que cette crainte ait été balayée alors qu'il parle avec fierté de ce qu'est devenue l'«École de Montréal »4.

Après un premier virage amorcé, c'est Nicolas Bernier qui reprendra la barre de la direction artistique de 2008 à 2011. La relève montréalaise ayant toujours eu une place importante au sein de la programmation, Bernier, issu de la deuxième génération de l'«École de Montréal », continue dans cette perspective. Les compositeurs québécois et canadiens sont de plus en plus représentés et sont même majoritaires chaque saison. Réseaux commence à emprunter les routes du Québec et diffuse hors de la métropoles. La saison anniversaire 2011-2012 est la première sous la direction artistique du compositeur Louis Dufort. Également issu du bassin de compositeurs montréalais, il commence son mandat avec une saison charnière et, pour ce faire, il s'entoure d'un comité artistique constitué de quinze compositeurs de la relève ou établis. Dufort présente cette programmation spéciale comme étant née de «choix musicaux éclectiques». Ces choix sont représentatifs de la diversité et des différentes tangentes de la musique électroacoustique.

\section{Comité artistique $20^{\mathrm{e}}$ anniversaire}

Martin Bédard, Nicolas Bernier, Christian Calon, Chantal Dumas, Gilles Gobeil, Monique Jean, France Jobin, Chantal Laplante, Martin Leclerc, Emmanuel Madan, Martin Marier, Robert Normandeau, Sylvain Pohu, Hélène Prévost, Patrick St-Denis 


\section{... immersive}

La vingtième saison de Réseaux des arts médiatiques a offert au public montréalais un déferlement d'événements: quatre concerts et trois conférences lors de la huitième édition d'Aкousma, six concerts de la série Électrochoc en coproduction avec le Conservatoire de musique de Montréal, deux conférences et deux concerts en coproduction avec le Centre interdisciplinaire de recherche en musique, médias et technologie (СIRмmT), le Cégep Saint-Laurent et la Fédération des associations de musiciens éducateurs du Québec (FAMEQ). Cette année festive a donné à nouveau l'occasion de diffuser une partie de la programmation hors Québec avec les concerts de la série Akousma_ex à Troy (NY) et au festival Electric Fields d'Ottawa. Ces événements ont présenté autant de nouveaux invités que de figures marquantes pour l'organisme tels Francis Dhomont, l'un des pères de cette «École de Montréal», et Annette Vande Gorne, une «grande inspiration » ${ }^{6}$.

Les concerts de chacune des séries ont proposé à l'auditeur de vivre son expérience d'écoute comme bon lui semblait: le grand explorateur allongé sur des coussins ou le contemplateur préférant encore un mode d'écoute plus traditionnel sur une chaise. Dans la pure tradition acousmatique ou avec des atmosphères lumineuses créées pour chacune des œuvres, de la douce ambiance colorée aux pulsions du stroboscope, Réseaux des arts médiatiques a offert une expérience musicale que seul le concert peut donner en présentant les branches et les sous-branches de la musique électroacoustique.

\section{La programmation de la saison $20^{\mathrm{e}}$ anniversaire}

ÉLEctrochoc Conservatoire de musique de Montréal

(Coproduction avec le смм)

22 septembre 2011 • Igor Lintz-Maues (AUT-BR)

10 novembre 2011 - Alain Savouret (FR)

$1^{\text {er }}$ décembre 2011 - Robert Normandeau (QC)

8 décembre 2011 • Christian Calon (FR/QC)

9 février 2012 - Nicolas Bernier (QC)

15 mars 2012 - Yves Daoust (QC)

EMPAC Troy, NY

7 octobre 2011 • Pierre-Yves Macé (FR), France Jobin (QC),

Louis Dufort (QC), Horacio Vaggione (FR/AR)

\section{ELECTRIC FIELDS Ottawa}

24 novembre 2011 - Jean-François Laporte (QC), Martin Bédard (QC), Roger Tellier-Craig (Qc)
6. Programme du concert Akousma@ CIRMMT (2012), MMR, Montréal (4 avril). 
7. Concert Akousma live@cIRmmt (jeudi 5 avril 2012).
Aкousma 8 - Usine C, Montréal

\section{Concerts}

12 octobre 2011 • Pierre-Yves Macé (FR), Roger Tellier-Craig (QC)

13 octobre 2011 • Hélène Prévost (QC), Stephan Mathieu (DE)

14 octobre 2011 - France Jobin (QC), Robert Hampson (GB)

15 octobre 2011 - Marc Behrens (DE), Horacio Vaggione (FR/AR)

Conférences

13 octobre 2011 • Horacio Vaggione, Université de Montréal

14 octobre 2011 - Marc Behrens, Université Concordia

14 octobre 2011 - Horacio Vaggione, Conservatoire de musique de Montréal

\section{RÉSEAUX_20 CIRMMT, Université McGill, Montréal}

En collaboration avec le Centre interdisciplinaire de recherche en musique, médias et technologie (CIRMMT), le Cégep Saint-Laurent et la Fédération des associations de musiciens éducateurs du Québec (FAMEQ).

\section{Concerts}

4 avril 2012 - Francis Dhomont (FR), Annette Vande Gorne (BE)

5 avril 2012 • Jean-François Denis (QC), Gilles Gobeil (QC), Robert Normandeau (QC)

\section{Conférences}

4 avril 2012 - Robert Normandeau : 20 ans de Réseaux

5 avril 2012 - Francis Dhomont: Francis Dhomont: Autour de l'"École de Montréal"

\section{... créative}

Réseaux des arts médiatiques est, depuis deux décennies, à l'écoute de son milieu, et cette période a été synonyme de changement, d'évolution. D’un point à l'autre, le décor a pris forme, a changé les perspectives. L'oreille de l'auditeur s'est tendue et est restée attentive aux sons proposés.

Nous laisserons le dernier mot à Jean-François Denis avec ses paroles passionnées alors qu'il nous parle de cette musique électroacoustique: «[...] il faut la présenter avec les meilleurs systèmes possibles parce que c'est une musique qui le mérite? ! » 